\title{
Análise do número de casos de hanseníase com incapacidade grau II nos estados brasileiros frente às metas mundiais
}

\author{
Analysis of the number of cases of leprosy with disability grade II in brazilian \\ states facing the world goals
}

1 Lorenna Jacob de Mendonça lorenjacobm@yahoo.com

1 Marina Valéria Mendes

2 Angela Schächter Guidoreni

1 Luiza De Paula Faustino

1 Rachel Valente Ramos

1 Graduanda do curso de Medicina do Centro Universitário de Volta Redonda - UniFOA.

2 Mestrado Interdisciplinar em Educação e Saúde. Docente do curso de Medicina do Centro Universitário de Volta Redonda - UniFOA

\section{Resumo}

O Brasil, em 2018, ocupava o segundo lugar na relação de países com maior número de casos de hanseníase no mundo, o que exigiu a adoção de estratégias para combater a doença, reduzir as incapacidades e o estigma a ela relacionado. Nesse sentido, propomos um estudo com base nos dados disponibilizados pelo Ministério da Saúde, através do DATASUS, com objetivo de analisar as taxas de incidência de Hanseníase com incapacidade grau II, no período de 2016 a 2019, nos estados brasileiros, considerando o número total e os dados referentes à faixa etária de 0 a 14 anos, separadamente; avaliar se houve redução no número de casos e se o Brasil está em consonância com as metas estabelecidas pela Organização Mundial de Saúde de reduzir a taxa de casos novos com grau 2 de incapacidade física para menos de 1 caso por 1.000.000 habitantes e zerar o número de casos com grau 2 em crianças, no período de 2016 a 2020. Em relação ao número total da incidência de casos de hanseníase com incapacidade grau II no intervalo entre os anos de 2016 e 2019, os resultados encontrados mostraram uma redução em quatro dos estados brasileiros; permanência em dois estados; e aumento no restante do país. Já na faixa etária de 0 a 14 anos, em relação à incidência de casos com avaliação de incapacidade grau II, observou-se uma diminuição em oito estados; inalteração em outros sete e o aumento nos onze restantes. Esses resultados mostram que o Brasil, no período de 2016 a 2019, se manteve distante das metas propostas pela Organização Mundial de Saúde.

\section{Palavras-chave:}

Hanseníase. Incidência. Doença de notificação compulsória. Organização Mundial de Saúde. Sistema Único de Saúde.

\begin{abstract}
In 2018, Brazil ranked second in the list of countries with the highest number of leprosy cases in the world, which required the adoption of strategies to deal with the disease, reduce disabilities and the stigma related to it. In this case, we propose a study, based on data available by the Ministry of Health, through DATASUS, with the objective of analyzing the incidence rates of Hansen's disease with grade II disability, in the period from 2016 to 2019, in the Brazilian states, considering the total amount and data referring to the age group from 0 to 14 years, separately; evaluating whether it has been a reduction in the number of cases and if Brazil is in line with the goals established by the World Health Organization to reduce the rate of new cases with grade 2 physical disability to less than 1 case per 1,000,000 inhabitants and reset the number of cases with grade 2 in children, in the period from 2016 to 2020. Regarding the total number of leprosy cases with grade II disability in the interval between 2016 and 2019, the results found showed a reduction in four of the Brazilian states; a permanence in two states; and an increase in the rest of the country. In the age group from 0 to 14 years, in relation to the incidence of cases with grade II disability assessment, there was a decrease in eight states; unchanged in another seven and an increase in the remaining eleven. These results show that Brazil, in the period from 2016 to 2019, remained distant from the goals proposed by the World Health Organization.
\end{abstract}

\section{Keywords:}

Leprosy. Incidence. Compulsory reporting disease. World Health Organization. Unified Health System.

Como você deve citar?

MENDONÇA, Lorenna Jacob de et al. Análise do número de casos de hanseníase com incapacidade grau II nos estados brasileiros frente às metas mundiais. Cadernos UniFOA, Volta Redonda (RJ), v. 16, n. 47, p. 113 - 119, dez, 2021. 
A hanseníase é uma doença infecciosa, transmissível e de caráter crônico que ainda persiste como problema de saúde pública no Brasil (BRASIL, 2008). No mundo, foram reportados à Organização Mundial da Saúde (OMS) 208.619 casos novos da doença, em 2018. Desses, 30.957 ocorreram na região das Américas e 28.660 (92,6\% do total das Américas) foram notificados no Brasil. Do total de casos novos diagnosticados no país, 1.705 (5,9\%) ocorreram em menores de 15 anos. Quanto ao Grau de Incapacidade Física (GIF), entre os 24.780 (86,5) avaliados no diagnóstico, 2.109 (8,5\%) apresentaram deformidades visíveis (SAÚDE, 2019).

A eliminação da hanseníase como problema de saúde pública foi alcançada em 2000 em escala mundial e, até 2005, na maioria dos países, no entanto, em alguns, a exemplo do Brasil, a agenda de eliminação da doença no âmbito subnacional ainda está inconclusa e continuará a ser aplicada nos próximos anos. Tal fato se deve à permanência de alguns desafios: a continuação do atraso na detecção de novos pacientes, a persistência da discriminação de pessoas afetadas pela afecção e o impacto limitado na sua transmissão. Sendo assim, a Estratégia Global para Hanseníase 2016 - 2020 teve como propósito promover maior integração no âmbito nacional entre serviços de hanseníase e outros serviços, nos níveis primários e de referência, a fim de se combater a doença, as incapacidades e o estigma ligado a ela, que ocorre desde tempo imemoráveis. Para isso, a meta é reduzir a taxa de casos novos com grau 2 de incapacidade física para menos de 1 caso por 1.000 .000 habitantes e zerar o número de casos com grau 2 em crianças (ORGANIZAÇÃO MUNDIAL DA SAÚDE, 2016).

Considerando que se trata de uma doença conhecida há mais de 400 anos e com tratamento implementado há cerca de 30 anos, era de se esperar que as taxas de incidência de hanseníase tivessem reduzido. No entanto, o Brasil assumiu a segunda posição entre os países com maior número de casos em 2018, justificando a necessidade de se pesquisar de forma mais detalhada os dados epidemiológicos dessa doença no Brasil.

Diante do exposto, torna-se relevante desenvolver um estudo cujos objetivos são: analisar as taxas de incidência de Hanseníase com incapacidade grau II, no período de 2016 a 2019, nos estados brasileiros, considerando o número total e os dados referentes à faixa etária de 0 a 14 anos, separadamente; avaliar se houve redução no número de casos e se o Brasil está em consonância com as metas estabelecidas pela Organização Mundial de Saúde.

\section{METODOLOGIA}

Trata-se de um estudo epidemiológico observacional descritivo em que foi realizada uma análise exploratória, no período de 2016 a 2019 (ano mais recente disponível nas bases de dados utilizadas), voltada para a magnitude dos casos de hanseníase como problema de saúde pública em todo o país.

Os dados utilizados foram provenientes do DATASUS/MS (Banco de dados do Sistema Único de Saúde), em que se selecionou separadamente cada estado brasileiro, clicando em "Ano Diagnóstico", "Não ativa", "Frequência" e "Grau II", respectivamente, nas opções "Linha", "Coluna", "Conteúdo" e "Aval Incap Notif", entre os períodos disponíveis de 2016 a 2019 e, posteriormente, fazendo uma nova busca, acrescentando "0-14 anos" na "Faixa Etária Hans". Vale ressaltar que os "períodos disponíveis" correspondem aos anos de notificação dos casos e que esse sistema afirma a possibilidade de atualizações nos anos de 2016 a 2019, mas apresenta como última data de disponibilização de dados a de 10/2020. Esses dados foram analisados de forma bruta, não levando em consideração a proporção a cada 1.000.000 habitantes. 
A pesquisa foi realizada nas plataformas PubMed, Google acadêmico e Scielo, por meio das palavras-chaves: "hanseníase", "incidência", "doença de notificação compulsória", "organização mundial de saúde", "sistema único de saúde", tendo sido selecionados os artigos que tratavam de hanseníase de modo geral (história, conceito, diagnóstico, tratamento) e das estratégias globais para hanseníase 2016-2020. Por fim, foram incluídos, para compor a fundamentação teórica, 9 referências.

\section{REVISÃO BIBLIOGRÁFICA}

A hanseníase é uma doença infecciosa, crônica, causada por uma bactéria denominada mycobaterium leprae, que atinge pessoas de todas as idades, principalmente aquelas na faixa etária economicamente ativa, tendo assim grande importância para a saúde pública, devido à sua magnitude e seu alto poder incapacitante. A transmissão se dá por meio de uma pessoa doente, sem tratamento, que, pelas vias áreas superiores, elimina o bacilo para o meio exterior, infectando outras pessoas suscetíveis. (BRASIL, 2001).

O diagnóstico é realizado por meio do exame dermatoneurológico, com o objetivo de se identificar lesões ou áreas de pele com alteração de sensibilidade e/ou comprometimento de nervos periféricos (BRASIL, 2001). Tal doença possui tratamento e cura. Porém se, no momento do diagnóstico, o paciente já apresentar alguma deformidade física instalada, esta pode ficar como sequela permanente no momento da alta. Esse dado reforça a importância do diagnóstico precoce e do início imediato do tratamento adequado para a prevenção das incapacidades físicas que a evolução da doença pode causar (EIDT, 2004).

O Grau de Incapacidade Física (GIF) é o indicador da existência de perda sensitiva e/ou deformidade visível decorrente da lesão neural. Como indicador epidemiológico, ele é utilizado na avaliação dos programas de vigilância em hanseníase, ou seja, determinando a precocidade do diagnóstico e o sucesso das atividades de encerramento da transmissão da doença. A classificação se dá em 0, 1 e 2 de GIF, sendo, quanto maior o número, mais comprometido é o indivíduo. Enquadram-se como GIF 2, algumas das deficiências descritas a seguir: lagoftalmo, ectrópio, opacidade corneana central, atrofia muscular, mão e/ou pé caído, contraturas, feridas tróficas e/ou traumáticas nos pés e/ou nas mãos (SILVA et al., 2018).

Os primeiros casos da doença no país foram notificados no ano de 1600 e, desde a introdução da poli quimioterapia (PQT), há cerca de três décadas, a carga de hanseníase no mundo diminuiu consideravelmente, momento em que passou a ser tratada em hospitais e no âmbito da atenção primária à saúde (BRASIL, 1989; SAÚDE, 2019).

Com objetivo de racionalizar o processo de coleta e transferência de dados de doença e agravos, foi implantado, em 1993, o Sistema de Informação de Notificação de Agravos Nacionais (SINAN), porém, somente em 1995, seus bancos de dados foram estruturados, tornando a hanseníase uma das doenças de notificação compulsória de tal sistema (BORBA, 2015).

Essa moléstia, interpretada até mesmo como castigo divino, representa, desde os mais remotos tempos até os dias atuais, verdadeiro estigma social. Da crença de sua contagiosidade e de sua incurabilidade nasceu o medo de contraí-la e sofrer todos os males que ela representa, dentre os quais, o de ficar estigmatizado. Daí a repulsa que ela causava e o rigor social imposto aos seus portadores no passado (EIDT, 2004).

Diante de tal contexto histórico e epidemiológico, a hanseníase está inserida na agenda internacional e, dentre os compromissos mundialmente assumidos, a doença está contemplada no Objetivo 3 
de Desenvolvimento Sustentável (ODS) da Organização das Nações Unidas (ONU), que visa promover o bem-estar e uma vida saudável, com a meta de combater diversas doenças transmissíveis e tropicais negligenciadas até o ano de 2030. Além disso, a OMS traz a Estratégia Global para Hanseníase 20162020, que tem como meta reduzir a taxa de casos novos com grau 2 de incapacidade física para menos de 1 caso por 1.000 .000 habitantes e zerar o número de casos com grau 2 em crianças (SAÚDE, 2016).

No âmbito nacional, o Ministério da Saúde elaborou a Estratégia Nacional para Enfrentamento da Hanseníase 2019-2022. 0 documento tem como visão um Brasil sem hanseníase. A Estratégia Nacional se baseia na Estratégia Global e tem como objetivo geral reduzir a carga da doença no país ao fim de 2022, e possui as seguintes metas: 1 ) reduzir para 30 o número total de criança com grau 2 de incapacidade física; 2) reduzir para 8,83/1 milhão de habitantes a taxa de pessoas com grau 2 de incapacidade física; e 3) implantar, em todas as Unidades da Federação, canais para registro de práticas discriminatórias às pessoas acometidas pela hanseníase e seus familiares (BRASIL, 2019).

\section{RESULTADOS E DISCUSSÃO}

Descrevem-se a seguir, os resultados obtidos através dos dados coletados no DATASUS, conforme o método descrito.

Na tabela abaixo apresentam-se os dados brutos para os números de casos com incapacidade grau II na notificação, em 2016, 2017, 2018 e 2019, e os números de casos com incapacidade grau II na notificação na faixa etária de 0 a 14 anos (parcial), em 2016, 2017, 2018 e 2019.

Tabela 1: Número de casos de hanseníase com incapacidade grau II (total) e número de casos de hanseníase com incapacidade grau II na faixa etária de 0-14 anos (parcial) nos anos de 2016 a 2019.

\begin{tabular}{cccc}
\hline \multicolumn{4}{c}{ Número de casos incapacidade grau II } \\
\hline 2016 & 2017 & 2018 & 2019
\end{tabular}

\begin{tabular}{|c|c|c|c|c|c|c|c|c|}
\hline Estados & Total & Parcial & Total & Parcial & Total & Parcial & Total & Parcial \\
\hline Amazonas & 72 & 3 & 70 & 1 & 65 & 0 & 62 & 2 \\
\hline Acre & 7 & 0 & 17 & 0 & 22 & 1 & 14 & 0 \\
\hline Rondônia & 31 & 0 & 53 & 0 & 89 & 2 & 48 & 1 \\
\hline Amapá & 7 & 0 & 10 & 1 & 13 & 1 & 10 & 1 \\
\hline Tocantins & 131 & 3 & 176 & 5 & 208 & 3 & 199 & 1 \\
\hline Maranhão & 294 & 2 & 316 & 13 & 355 & 12 & 362 & 13 \\
\hline Piauí & 68 & 1 & 98 & 1 & 93 & 1 & 61 & 1 \\
\hline Ceará & 152 & 4 & 160 & 3 & 200 & 8 & 174 & 1 \\
\hline $\begin{array}{l}\text { Rio Grande do } \\
\text { Norte }\end{array}$ & 18 & 0 & 22 & 1 & 23 & 0 & 19 & 0 \\
\hline
\end{tabular}




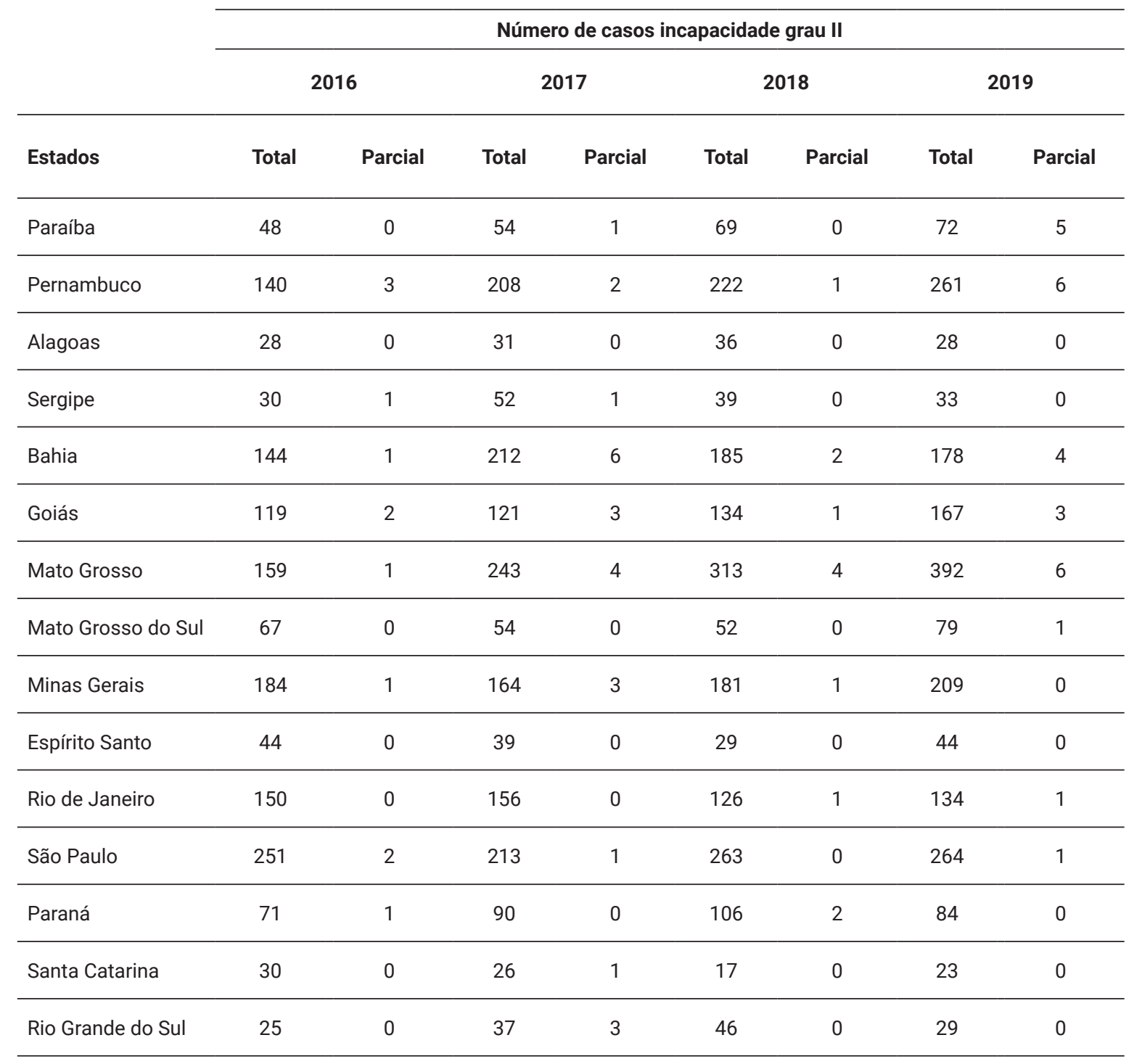

Fonte: DATASUS, 2020

A análise dos dados mostrou que as incidências de casos com avaliação de incapacidade grau II na notificação, comparadas nos anos de 2016 e 2019, sofreram redução apenas em quatro estados: Amazonas, Piauí, Rio de Janeiro e Santa Catarina, em que as taxas encontradas foram $13,3 \% ; 10,29 \%$; 10,6\%; e 23,33\%, respectivamente. Nos estados de Alagoas e Espírito Santo, houve a permanência da incidência. Já no restante do país, observa-se um aumento importante, sendo: 11,11\%, em Roraima; $100 \%$, no Acre; $54,83 \%$, em Rondônia; $42,85 \%$, no Amapá; $33,2 \%$, no Pará; $51,9 \%$, em Tocantins; $23,12 \%$, no Maranhão; $14,47 \%$, no Ceará; 5,55\%, no Rio Grande do Norte; 50\%, na Paraíba; $86,42 \%$, em Pernambuco; $10 \%$, em Sergipe; $23,61 \%$, na Bahia; $40,33 \%$, em Goiás; $146,54 \%$, no Mato Grosso; $17,91 \%$, no Mato Grosso do Sul; 13,58\%, em Minas Gerais; 5,17\%, em São Paulo; 18,3\%, no Paraná e 16\%, no Rio Grande do Sul.

Em relação às incidências de casos com avaliação de incapacidade grau II na notificação entre a faixa etária de 0 a 14 anos comparadas nos anos de 2016 e 2019, houve a redução em oito estados (100\%, em Roraima, Sergipe, Minas Gerais e Paraná; 33,33\%, no Amazonas; $66,66 \%$, em Tocantins; $50 \%$, em São Paulo e 75\%, no Ceará), permanência em outros sete (Acre, Piauí, Rio Grande do Norte, Alagoas, Espírito Santo, Santa Catarina e Rio Grande do Sul) e aumento nos onze restantes (surgimento de um caso em Mato Grosso do Sul, Rio de Janeiro, Rondônia, Amapá e Goiás; três em Pernambuco, Pará e Bahia; cinco em Paraíba e Mato Grosso; e onze em Maranhão). 
Ao término da observação desses dados, fica evidente que o Brasil não está contribuindo para a meta proposta pela OMS de reduzir a taxa de casos novos com grau 2 de incapacidade física para menos de 1 caso por 1.000.000 habitantes e zerar o número de casos com grau 2 em crianças. Isso porque, analisando os dados de forma bruta, 20 dos seus 26 estados tiveram aumento no número de casos com incapacidade funcional grau II, notificados em 2019, em relação a 2016 e apenas o estado do Espírito Santo permaneceu sem nenhum caso de hanseníase com incapacidade funcional grau II na faixa etária de 0 a 14 anos, entre os anos de 2016 e 2019.

O estudo dos dados nos leva a concluir também que a hanseníase ainda é uma doença negligenciada no Brasil, embora o Sistema Único de Saúde (SUS) possibilite o acesso ao diagnóstico, tratamento e tenha um sistema de informação atuante. Outro ponto importante dessa análise é perceber que as estratégias adotadas são insuficientes, o que vem ao encontro do alarde feito pelos órgãos de saúde, mundiais e nacionais, para a necessidade de uma postura mais rígida em relação ao enfrentamento da doença.

Diante disso, revela-se a necessidade de maior empenho na descoberta de novos casos, o que implica em maior suspeição de hanseníase, confirmação diagnóstica, tratamento e investigação dos contactantes do caso constatado. Para o cumprimento de todas essas etapas, é imprescindível atribuir responsabilidades e, ao mesmo tempo, capacidade a todos os envolvidos no processo de diagnóstico-cura. Dessa forma, os profissionais de saúde devem estar aptos para reconhecer os sinais e sintomas da doença, tratá-la e produzir medidas de promoção de saúde; as unidades de saúde devem se organizar, a fim de garantir o acesso da população aos serviços de promoção de saúde, prevenção de enfermidades, recuperação, reabilitação e acompanhamento/avaliação do cuidado; e a população deve estar ciente dos sinais e sintomas da doença, que há cura e que o tratamento é ofertado pela unidade de saúde de sua comunidade.

Essas medidas visam ao empoderamento tanto do doente quanto do profissional de saúde frente à doença, que juntos podem atuar em sinergia para o combate à hanseníase e alcance das metas propostas.

\section{REFERÊNCIAS}

BRASIL. Ministério da Saúde. Controle da hanseníase: uma proposta de integração ensino-serviço. Rio de Janeiro: DNDS/NUTES, 1989.

BRASIL. Ministério da Saúde. Secretaria de Vigilância em Saúde. Departamento de Doenças de Condições Crônicas e Infecções Sexualmente Transmissíveis. Estratégia Nacional para Enfrentamento da Hanseníase 2019-2022. Brasília: Ministério da Saúde, 2019.

BRASIL. Ministério da Saúde. Secretaria de Atenção à Saúde. Departamento de Atenção Básica. Caderno de Atenção Básica $n^{\circ}$ 21. Vigilância em Saúde: Dengue, Esquistossomose, Hanseníase, Malária, Tracoma e Tuberculose. 2. ed. rev. Brasília (DF): Ministério da Saúde; 2008.

BRASIL. Ministério da Saúde. Secretaria de Políticas de Saúde. Departamento de Atenção Básica. Hanseníase: Atividades de controle e manual de procedimentos/ área técnica de dermatologia. Brasília (DF): Ministério da Saúde; 2001.

BORBA, S. M. L. Vigilância epidemiológica da hanseníase na atenção básica: o caso do município de Itaboraí, região metropolitana do Rio de Janeiro. Rio de Janeiro, outubro de 2015. Disponivel em: http:// 
www6.ensp.fiocruz.br/repositorio/sites/default/files/arquivos/Vigil\%C3\%A2nciaHansen\%C3\%ADase. pdf. Acesso em: 01 dez. 2020.

EIDT, LETÍCIA MARIA. Breve história da hanseníase: sua expansão do mundo para as Américas, o Brasil e o Rio Grande do Sul e sua trajetória na saúde pública brasileira. Saude soc., São Paulo, v. 13, n. 2, p. 76-88, Aug. 2004 . Disponível em: http://www.scielo.br/scielo.php?script=sci_arttext\&pid=S0104$12902004000200008 \&$ Ing=en\&nrm=iso. Acesso em: 01 dez. 2020.

ORGANIZAÇÃO MUNDIAL DA SAÚDE. Estratégia mundial de eliminação da hanseníase 2016-2020: Acelerar a ação para um mundo sem hanseníase. 2016. Disponível em: https://apps. who.int/iris/bitstream/ handle/10665/208824/9789290225201-pt.pdf. Acesso em: 11 nov. 2020.

ORGANIZAÇÃO MUNDIAL DA SAÚDE. Global leprosy update, 2018: moving towards a leprosy free world. Weekly Epidemiological Record, Genebra, n. 94, p. 389-412, 30 ago. 2019. Disponível em: https://apps. who. int/iris/bitstream/ handle/10665/326775/WER9435-36-en-fr.pdf?ua=1. Acesso em: 9 out. 2020.

SILVA, et al. Universidade Federal de Santa Catarina. Centro de Ciências da Saúde. Núcleo Telessaúde Santa Catarina. Abordagem da Hanseníase na atenção básica. Florianópolis: CCS/UFSC, 2018. 